\title{
Bottom-to-top decomposition of time series by smoothness-controlled cubic splines: Uncovering distinct freezing-melting dynamics between the Arctic and the Antarctic
}

\author{
Imre M. Jánosi $\odot^{*}$ and Ágnes Baki॰ \\ Department of Physics of Complex Systems, Eötvös Loránd University, Pázmány Péter Street 1/A, 1117 Budapest, Hungary \\ Marcus W. Beims $\odot^{\dagger}$ \\ Departamento de Física, Universidade Federal do Paraná, 81531-990 Curitiba, Brazil \\ Jason A. C. Gallas ${ }^{\dagger}$ \\ Instituto de Altos Estudos da Paraíba, Rua Silvino Lopes 419-2502, João Pessoa 58039-190, Brazil \\ and Complexity Sciences Center, 9225 Collins Avenue, Surfside, Florida 33154, USA
}

(Received 3 June 2020; accepted 7 September 2020; published 7 October 2020)

\begin{abstract}
The classical methods of identifying significant slow components (modes) in a strongly fluctuating signal usually require strict stationarity. A notable exception is the procedure called empirical mode decomposition (EMD), which is designed to work well for nonstationary and nonlinear (quasiperiodic) time series. However, EMD has some well-known limitations such as the end divergence effect, mode mixing, and the general problem of interpreting the modes. Methods to overcome these limitations, such as ensemble EMD or complete ensemble EMD with adaptive noise, promise an exact reconstruction of the original signal and a better spectral separation of the intrinsic mode functions (IMFs). All these variants share the feature that the decomposition runs from the top to the bottom: The first few IMFs represent the noise contribution and the last is a long-term trend. Here we propose a decomposition from the bottom to the top, by the introduction of smoothness-controlled cubic spline fits. The key tool is a systematic scan by cubic spline fits with an input parameter controlling the smoothness, essentially the number of knots. Regression qualities are evaluated by the usual coefficient of determination $R^{2}$, which grows monotonically when the number of knots increases. In contrast, the growth rate of $R^{2}$ is not monotonic: When an essential slow mode is approached, the growth rate exhibits a local minimum. We demonstrate that this behavior provides an optimal tool to identify strongly quasiperiodic slow modes in nonstationary signals. We illustrate the capability of our method by reconstruction of a synthetic signal composed of a chirp, a strong nonlinear background, and a large-amplitude additive noise, where all EMD-based algorithms fail spectacularly. As a practical application, we identify essential slow modes in daily ice extent anomalies at both the Arctic and the Antarctic. Our analysis demonstrates the distinct freezing-melting dynamics on the two poles, where apparently different factors are determining the time evolution of ice sheets. Thus, we believe that our methodology offers a competitive tool to identify modes in strongly fluctuating data and advances significantly the state of the art regarding the decomposition of nonlinear time series.
\end{abstract}

DOI: 10.1103/PhysRevResearch.2.043040

\section{INTRODUCTION}

The decomposition of nonlinear time series is a general problem pervading many branches of science, where a

\footnotetext{
*Also at Max Planck Institute for the Physics of Complex Systems, Nöthnitzer Straße 38, 01187 Dresden, Germany; imre.janosi@ttk.elte.hu

${ }^{\dagger}$ Also at Max Planck Institute for the Physics of Complex Systems, Nöthnitzer Straße 38, 01187 Dresden, Germany.

Published by the American Physical Society under the terms of the Creative Commons Attribution 4.0 International license. Further distribution of this work must maintain attribution to the author(s) and the published article's title, journal citation, and DOI. Open access publication funded by the Max Planck Society.
}

plethora of data is produced and recorded by advanced automated measuring systems. Thus, there is a great deal of interest in general methods capable of providing fruitful insight into the complex realm of time series. An interesting method extensively used during the past decades is the socalled empirical mode decomposition (EMD), which, along with its many variants, has gained popularity in several fields, especially in analyzing geophysical records [1-5], biomedical signals [6-9], coupled chaotic systems [10-12], economic and market data [13-15], and in other complex scenarios.

Decomposition of nonstationary, nonlinear, noisy signals is not well performed by traditional methods. Empirical mode decomposition provides a general tool for an adaptive signal decomposition into a finite number of narrowband intrinsic mode functions (IMFs), which are derived directly from the data [16-18]. The IMFs generated by the EMD algorithm are determined by the local extrema of the signal, and 

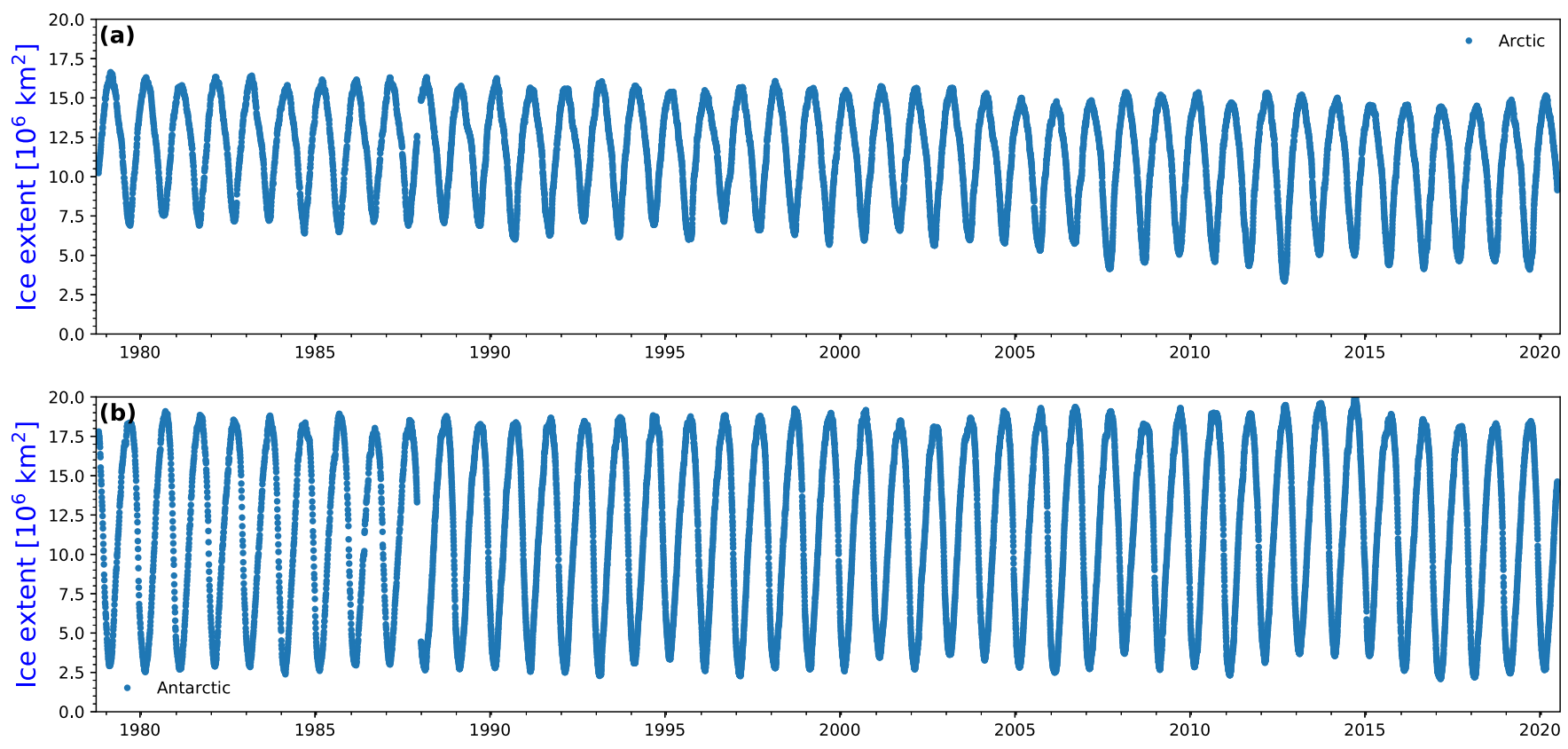

FIG. 1. Daily ice extent over (a) the Arctic and (b) the Antarctic in units of $10^{6} \mathrm{~km}^{2}$. Note missing data for 42 days at the end of 1988 .

traditional EMD uses a cubic spline for upper and lower envelope interpolation. Variants such as the ensemble EMD (EEMD) $[19,20]$ or complete ensemble EMD with adaptive noise (CEEMDAN) [21,22] are noise-assisted extensions of EMD, where the IMFs are constructed as a mean of an ensemble of decompositions where the original signal is decorated by different added white-noise series. These and other improvements can handle some shortcomings of the traditional EMD algorithm, e.g., mode mixing, where segments of a given characteristic frequency range are randomly distributed among two or more IMFs [23-25], or the problem of missing data points (see, e.g., [26]). Notably, the EMD algorithm works as a dyadic filter bank, that is, the mean frequency of a given IMF is approximately half of the previous one [26-28]. For this reason, the basic problem of all top-to-bottom decompositions is the interpretation: how to distinguish relevant IMFs and irrelevant IMFs in an efficient way.

Here we propose an alternative procedure providing a bottom-to-top decomposition. Similarly to EMD, this is an empirical method, purely data driven, and uses cubic spline fits. We have successfully validated the procedure on a series of synthetically constructed composite signals (see below and the Appendix). Specifically, we demonstrate the efficiency of the method on two real signals: daily sea ice extent over the Arctic and over the Antarctic as determined by satellite image processing (Fig. 1).

The original idea of smoothness-controlled cubic spline fits was explained by de Boor [29]. Smoothing splines are commonly used function estimates for a set of noisy observations with the goal of striking a balance between having a smooth curve and being close to the given data. The starting point to find an optimum is the smoothest possible curve, a straightline fit. When the data set is segmented with a growing number of knots, local cubic fits (with matching second derivatives at the knots) approach better and better the data set. A perfect fit is achieved by the natural cubic spline incorporating all the data points, with the consequence that the smooth fit can exhibit extreme waviness. The goodness of fit monotonically improves with an increasing number of knots; nevertheless, we have observed that the rate of improvement is not monotonic for composite signals. A large number of numerical experiments indicated that the growth rate of any correlationbased measure of goodness of fit slows down when the cubic spline approaches an essential slow mode (ESM), a given component in a synthetic signal. This observation is the key which permits a bottom-to-top decomposition of nonstationary nonlinear signals, as it is demonstrated in the remainder of the paper.

\section{DATA AND METHODS}

\section{A. Daily ice extent over both poles}

Figures 1(a) and 1(b) illustrate time series of daily ice extent over the Arctic and the Antarctic in the period between 26 October 1978 and 15 May 2020. These freely available data are collected and maintained by the U.S. National Ice and Snow Data Center [30]. During the first eight years of satellite observations, data for every second day were recorded, with a gap of 42 days at the end of 1988 [clearly visible in Fig. 1(b)]. Since the freezing-melting dynamics is apparently smooth enough, we used linear interpolation as a proxy for the missing data, resulting in the two evenly sampled time series of $N=15177$ days.

Climatological mean values for each calendar day were determined in the usual way [31]; averages over 36, 37, or 38 years were computed (depending on the available samples), including the 10 leap days (29 February). The results are plotted in Fig. 2 for both poles. The width of the orange band represents one standard deviation illustrating the larger variability of the Arctic ice cover around the annual cycle, as it is well known. Our starting point for the subsequent analysis is the ice extent anomaly, namely, the difference between a 

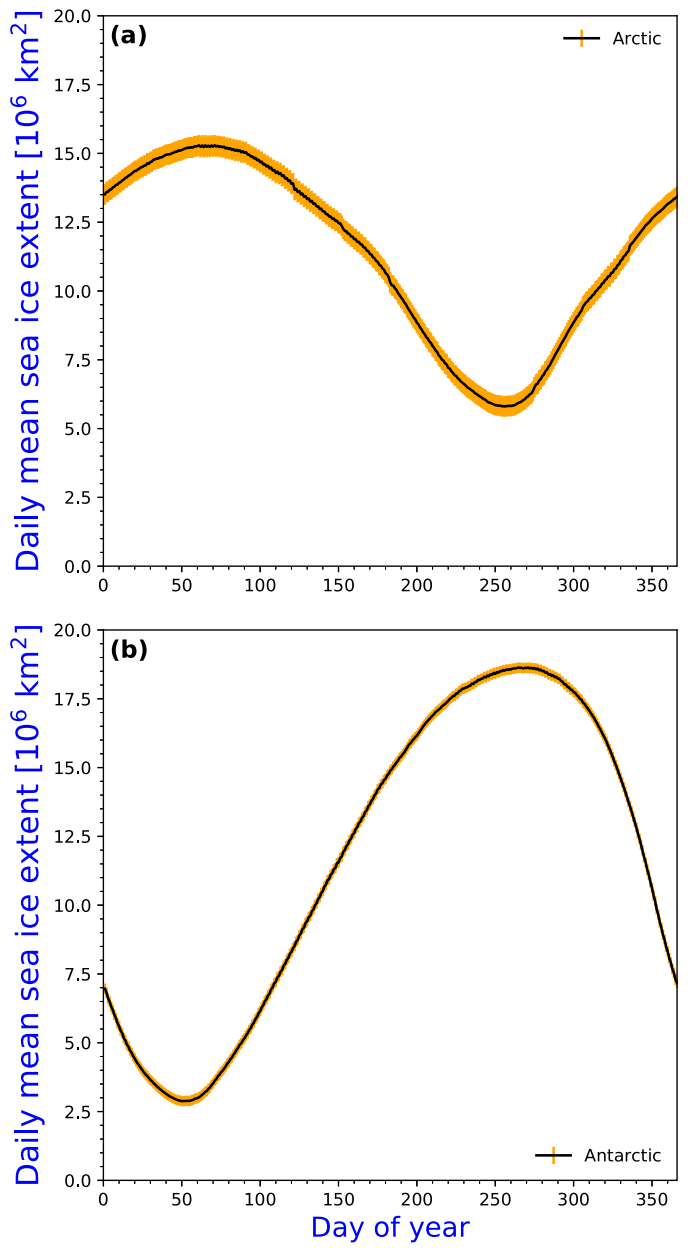

FIG. 2. Climatological mean of daily ice extent over (a) the Arctic and (b) the Antarctic in units of $10^{6} \mathrm{~km}^{2}$.

given observation and the climatological mean value on the particular calendar day. Note that the removal of climatological means does not affect long-term trends, e.g., when an average calculated over systematically decreasing values in consecutive years is subtracted, the tendency in the anomaly time series obviously remains the same.

\section{B. Smoothness-controlled cubic spline fits}

We implemented the PYTHON package CSAPS (version 0.11.0) developed by Prilepin for univariate, multivariate, and $n$-dimensional grid data approximations using cubic smoothing splines [32]. The methodology is based on the original algorithm of de Boor [29], where a smoothing spline $S$ with a smoothing parameter $p$ minimizes the sum of an error measure and a roughness measure

$$
p \sum_{i=1}^{N}\left[y_{i}-S\left(x_{i}\right)\right]^{2}+(1-p) \int S^{\prime \prime}(t)^{2} d t,
$$

where $\left\{x_{i}, y_{i}\right\}$ is the original data set and $S^{\prime \prime}$ denotes the second derivative. The smoothness parameter $p \in[0,1]$ has well-defined limits: $p=0$ belongs to a least-squares linear fit, while $p=1$ is the natural cubic spline.
A widely discussed point in the literature related to smoothing splines is the optimal choice of smoothing parameter $p$ [29,33-35]. For a recent survey see, e.g., Ref. [36]. Here we follow a different strategy by systematically evaluating spline fits in a wide range of smoothing parameter $p$, because we are interested in composite signals and not only in finding the optimal smoothing cubic spline. Our approach is illustrated in Fig. 3 with a synthetic signal $Y(t)$ composed from a chirp $C(t)$, a slow background trend $B(t)$, and an additive Gaussian noise $G(t)$. Our construction mimics the time range and amplitudes of the polar ice extent anomalies with the parameters

$$
C(t)=a \sin \left(\Omega t+\alpha t^{2}\right),
$$

with $a=0.3, \Omega=1.115 \times 10^{-3}$ day $^{-1}, \alpha=5 \times 10^{-7}$ day $^{-1}$, and $t=1,2, \ldots, N=15177$, and

$$
B(t)=b \sin \left(\omega_{1} t\right) \sin \left(\omega_{2} t\right),
$$

with $b=0.5, \omega_{1}=2.867 \times 10^{-4}$ day $^{-1}$, and $\omega_{2}=6.881 \times$ $10^{-4}$ day $^{-1}$. The additive Gaussian noise $G(t)$ has a unit amplitude and variance of $\frac{1}{2}$. The result for $Y(t)=C(t)+$ $B(t)+G(t)$ is plotted in Fig. 3(a) (black), where also the chirp component $C(t)$ (white) and the slow background $B(t)$ (yellow) are shown.

The quality of a smoothing cubic spline with a particular smoothing parameter $p$ is characterized by the usual coefficient of determination

$$
R^{2}=1-\frac{\sum_{i}\left[y_{i}-S\left(x_{i}\right)\right]^{2}}{\sum_{i}\left(y_{i}-\langle y\rangle\right)^{2}},
$$

where the nominator represents the sum of squared error of the fit and the denominator is the total variance of the signal. We are aware of the well-known limitations of correlation-based measures (see, e.g., [37]). However, this work is not aimed to analyze the occurrence of extreme cases such as enormous outliers and strongly correlated noise components.

By definition, the coefficient of determination $R^{2}$ grows monotonically when $p$ increases. Indeed, Fig. 3(b) illustrates well this relationship for our synthetic test signal $Y(t)$, where the results for 151 logarithmically placed $p$ values are shown, starting from $p_{1}=8 \times 10^{-15}$ and iterating as $p_{j}=1.24 p_{j-1}$, where $j=2,3, \ldots, 151$.

Notably, the growth rate of $R^{2}$ is not uniform, as demonstrated in Fig. 3(c), where the derivative $d\left(R^{2}\right) / d p$ is plotted. We have performed several numerical experiments with various synthetic signals composed of periodic, polynomial, chirplike [see Eq. (2)], piecewise linear, etc., and additive noise terms, obtaining the following general behavior. When the smoothing cubic spline approaches a fundamental mode, what we may call an essential slow mode, the growth rate of $R^{2}$ exhibits a local minimum. The first such minimum belongs to $\mathrm{ESM}_{1}$ of the smallest characteristic frequency range, the second minimum represents the superposition of the slowest and second-slowest modes $\mathrm{ESM}_{1}+\mathrm{ESM}_{2}$, and so on. This behavior provides the key for decomposing a nonstationary and nonlinear signal into ESMs with the following steps.

(1) Perform a scan over a wide enough range of smoothing parameter $p$ and determine $R^{2}$ for each cubic spline fit $S_{p}(t)$. (We recommend logarithmic spacing of $p$ values because, 

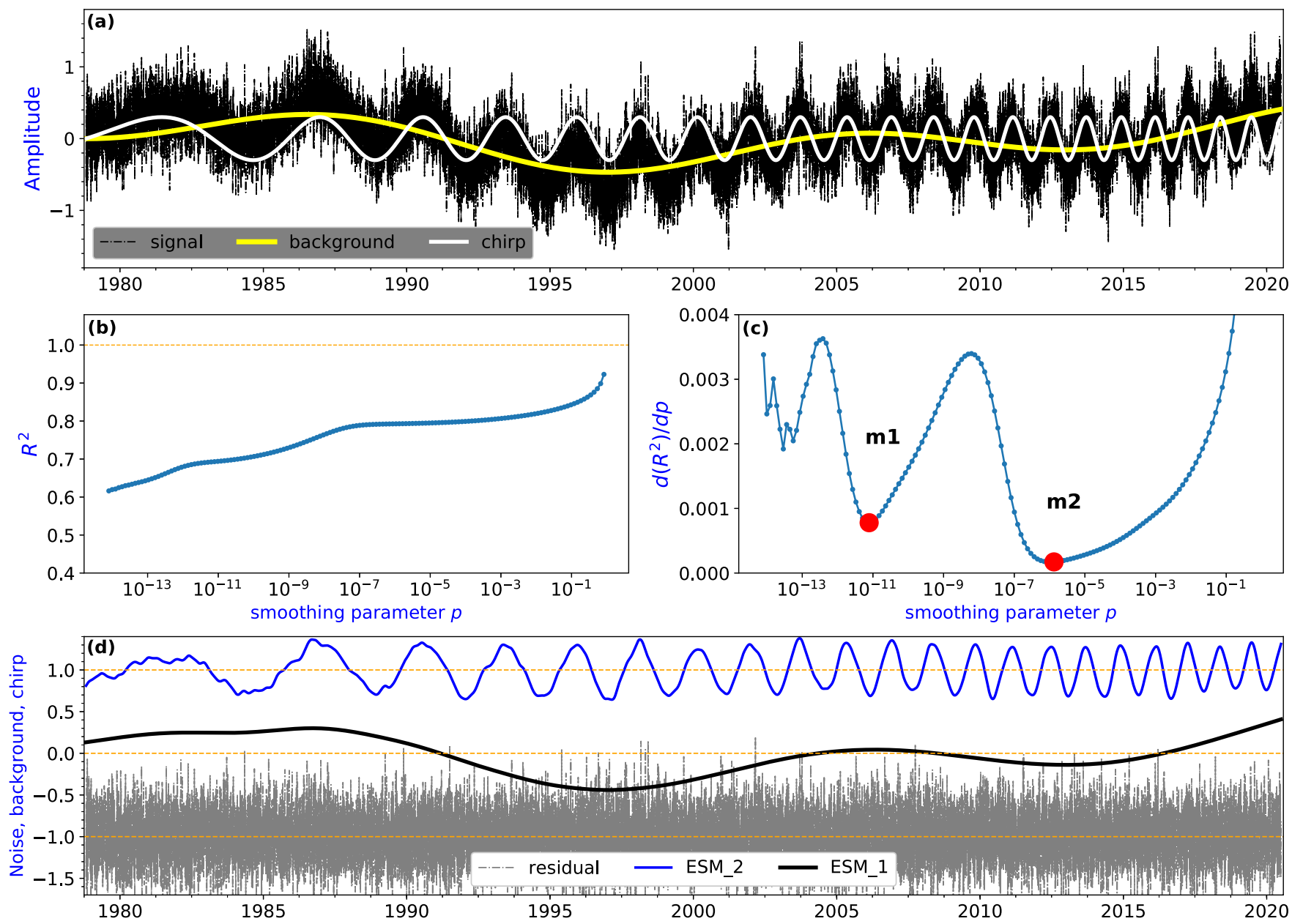

FIG. 3. (a) Synthetic signal (black) composed of a chirp (2) (white), a slow background (3) (yellow), and a Gaussian noise term with unit amplitude and variance of $\frac{1}{2}$. (b) Coefficient of determination $R^{2}$ [see Eq. (4)] as a function of smoothness parameter $p$. The horizontal axis is logarithmic. (c) Derivative of the curve in (b), $d\left(R^{2}\right) / d p$, as a function of $p$. The local minima indicated by red symbols identify the first and second ESMs; the corresponding smoothness parameters are $p_{1}=7.809 \times 10^{-12}$ and $p_{2}=1.331 \times 10^{-6}$. (d) Slow modes decomposed from the bottom to the top: $\operatorname{ESM}_{1}$ (black) is the cubic spline fit $S_{p_{1}}(t)$, while $\operatorname{ESM}_{2}$ (blue, shifted upward by 1.0) is the difference $S_{p_{2}}(t)-S_{p_{1}}(t)$. The residual (gray) is shifted downward by 1.0 .

in general, the growth of $R^{2}$ has more or less logarithmic tendencies.)

(2) Determine the local minima $\left\{p_{1}, p_{2}, p_{3}, \ldots, p_{M}\right\}$ of the empirical growth-rate function $d\left(R^{2}\right) / d p$.

(3) A proper decomposition of the original signal is iteratively given: The slowest mode $\mathrm{ESM}_{1}$ is the cubic spline fit $S_{p_{1}}(t)$ by $p_{1}$, the second slowest mode $\mathrm{ESM}_{2}$ is the difference of cubic spline fits $S_{p_{2}}(t)-S_{p_{1}}(t)$ by $p_{2}$ and $p_{1}$, the mode $\mathrm{ESM}_{3}$ is the difference of cubic spline fits $S_{p_{3}}(t)-S_{p_{2}}(t)$ by $p_{3}$ and $p_{2}$, etc. [note that $S_{p_{2}}(t)$ is already the superposition of $\mathrm{ESM}_{1}$ and $\left.\mathrm{ESM}_{2}\right]$.

Figure 3(d) illustrates the result of signal decomposition with two ESMs determined by the two local minima in Fig. 3(c) (red circles). A simple visual comparison with Fig. 3(a) demonstrates that both the chirp and the slow nonlinear background are nicely reconstructed with a little wriggling at the first third of the signal.

\section{Complete ensemble EMD with adaptive noise}

In order to compare the results of our bottom-to-top decomposition with results from the standard EMD, we used the
PYTHON package PYEMD developed by Lascuk [38]. Besides the original EMD and EEMD procedures, the package contains the CEEMDAN algorithm as described in Refs. [21,22]. Since the methodology is widely discussed in the literature $[21,22,39,40]$, we do not repeat the details here. The decomposition runs from the top to the bottom by using local maxima and minima as upper and lower envelopes at each step of the identification of an IMF.

The improved CEEMDAN algorithm [22] identified 12 IMFs for the synthetic test signal illustrated in Fig. 3(a). The first six IMFs represent noise terms with decreasing characteristic frequencies. The interesting part IMF-7, . ., IMF-12 is shown in Fig. 4. The top-to-bottom decomposition spectacularly fails to reconstruct the essential slow modes: The chirp part $C(t)$ is divided among IMF-7, IMF-8, and IMF-9; the background part $B(t)$ is divided among IMF-10 and IMF-11 (even the sum of them has serious flaws at the ends of the signal). The almost linear trend of small slope IMF-12 is a clear artifact of the procedure.

The decomposition of chirplike modes is a particularly demanding task where most classical methods fail. Since 

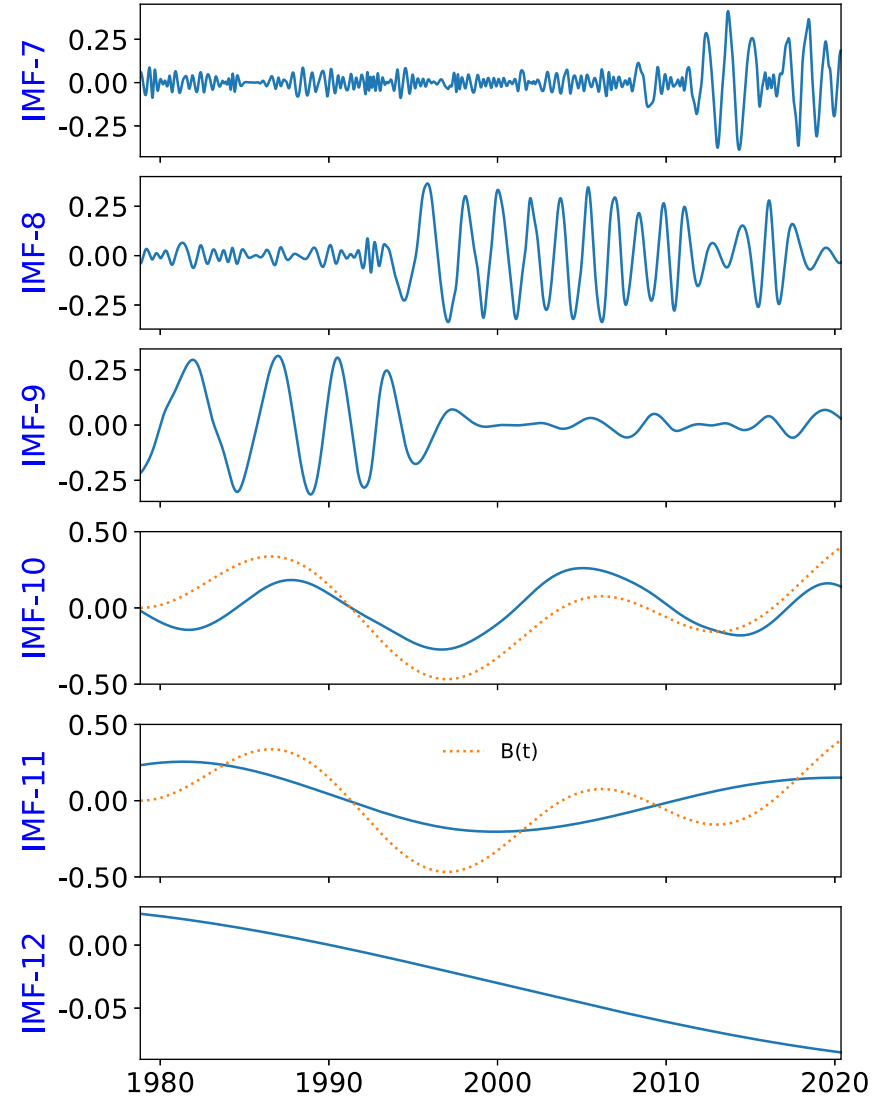

FIG. 4. Last six IMFs (blue lines) of the synthetic signal $Y(t)$ shown in Fig. 3(a) produced by the CEEMDAN algorithm. The chirp component $C(t)$ [see Eq. (2)] is apparently distributed among IMF-7, IMF-8, and IMF-9 in a strange way. Besides IMF-10 and IMF-11, orange dotted line illustrates the slow background component $B(t)$ given by Eq. (3).

practical signal processing often meets with the problem of smoothly changing internal frequencies, there are some recent proposals to handle such situations [41-43]. Our method seems to be by far the simplest.

\section{RESULTS FOR POLAR ICE EXTENT TIME SERIES}

As a representative example, we evaluate the two ice extent time series described in Sec. II A. Although these data sets are available at daily resolution, we have not found any publication where the whole records would have been evaluated. Probably the smoothness of the curves in Fig. 1 suggests that there is not much information in a daily datum; therefore, practically every analysis in the literature use monthly mean values as a starting point. Here we compare the results of our bottom-to-top decomposition method with the CEEMDAN procedure (see Sec. II C).

Figure 5 illustrates the results of the systematic scan by smoothing cubic spline fits as a function of $p$. As it is explained in Sec. II B, local minima on the growth-rate curves of the coefficient of determination $d\left(R^{2}\right) / d p$ identify the ESMs. Figure 5(b) exhibits three local minima for the Arctic time series, presumably a slow background tendency at $\mathrm{m} 1$, a small-amplitude slow oscillatory mode at $\mathrm{m} 2$, and a
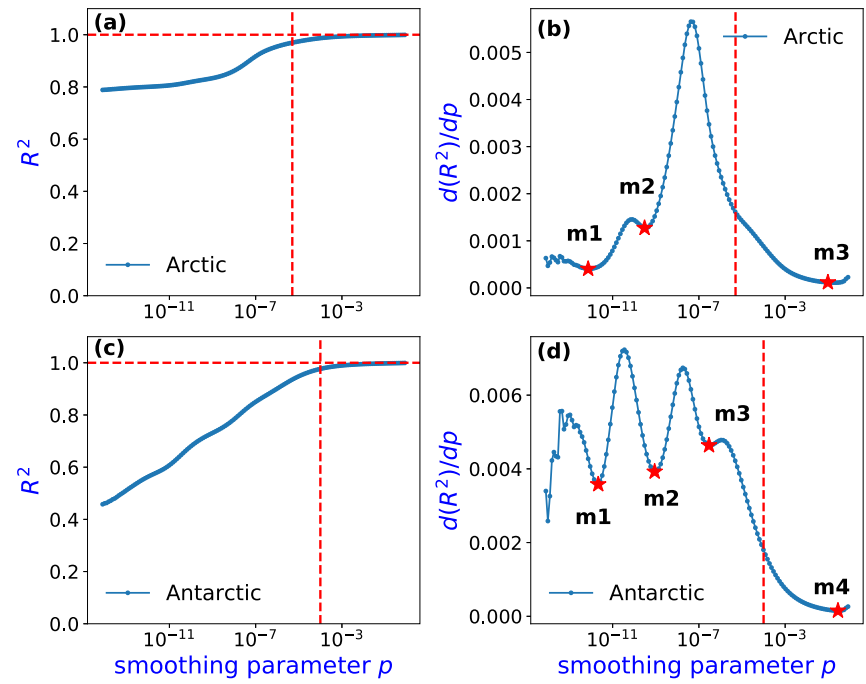

FIG. 5. Systematic analysis of the smoothing cubic spline fits for the Arctic and Antarctic ice extent anomaly time series. (a) Coefficient of determination $R^{2}$ [see Eq. (4)] as a function of smoothness parameter $p$ for the Arctic anomaly fits. The horizontal axis is logarithmic. (b) Derivative of the curve in (a), $d\left(R^{2}\right) / d p$, as a function of $p$. The local minima $\mathrm{m} 1$ and $\mathrm{m} 2$ indicated by red stars identify the first and second essential slow modes; the corresponding smoothness parameters are $p_{1}=7.327 \times 10^{-13}$ and $p_{2}=3.025 \times 10^{-10}$. The third local minimum $\mathrm{m} 3$ does not belong to a slow mode; it is an almost perfect fit for the original time series. The vertical dashed line at $p_{3}=5 \times 10^{-6}$ defines a slow mode (see the text). (c) and (d) Same as (a) and (b) but for the Antarctic anomalies. The first three minima are at $p_{1}=2.148 \times 10^{-12}, p_{2}=8.869 \times 10^{-10}$, and $p_{3}=2.953 \times 10^{-7}$. The vertical dashed line indicates $p_{4}=10^{-4}$.

large-amplitude higher-frequency mode at m3. However, at the smoothness parameter belonging to $\mathrm{m} 3$, the cubic spline has an almost perfect match with $R^{2} \approx 1.0$ [see Fig. 5(a)], indicating that all the high-frequency noise spikes are already incorporated in the fit. In order to have a smoother component, we can choose $p_{3}=5 \times 10^{-6}$ [vertical red dashed line in Fig. 5(b)]. This provides a very close match [see Fig. 5(a)], however with a dampened noise, as we will discuss below.

The same procedure for the Antarctic time series [Figs. 5(c) and 5(d)] locates four local minima; the situation at $\mathrm{m} 4$ is the same as for the Arctic m3. Instead of analyzing a perfect noisy fit, we estimate the highest-frequency ESM with a smoothness parameter $p_{4}=10^{-4}$ [vertical red dashed lines in Figs. 5(c) and 5(d)].

We might have expected that the two time series shown in Fig. 1 should be decomposed into similar ESMs (where ESM is the climatological mean plotted in Fig. 2 for both poles). In contrast, the results in Fig. 5 reflect very essential differences between the freezing-melting dynamics at the Arctic and Antarctic. Next we provide details and comparisons with the CEEMDAN decomposition.

\section{A. Arctic}

The ice extent anomaly time series for the Arctic is plotted in Fig. 6(a). Clearly, the removal of the climatological mean [Fig. 2(a)] from the original record [Fig. 1(a)] did not affect the long-term tendency, the well-documented dramatic ice 

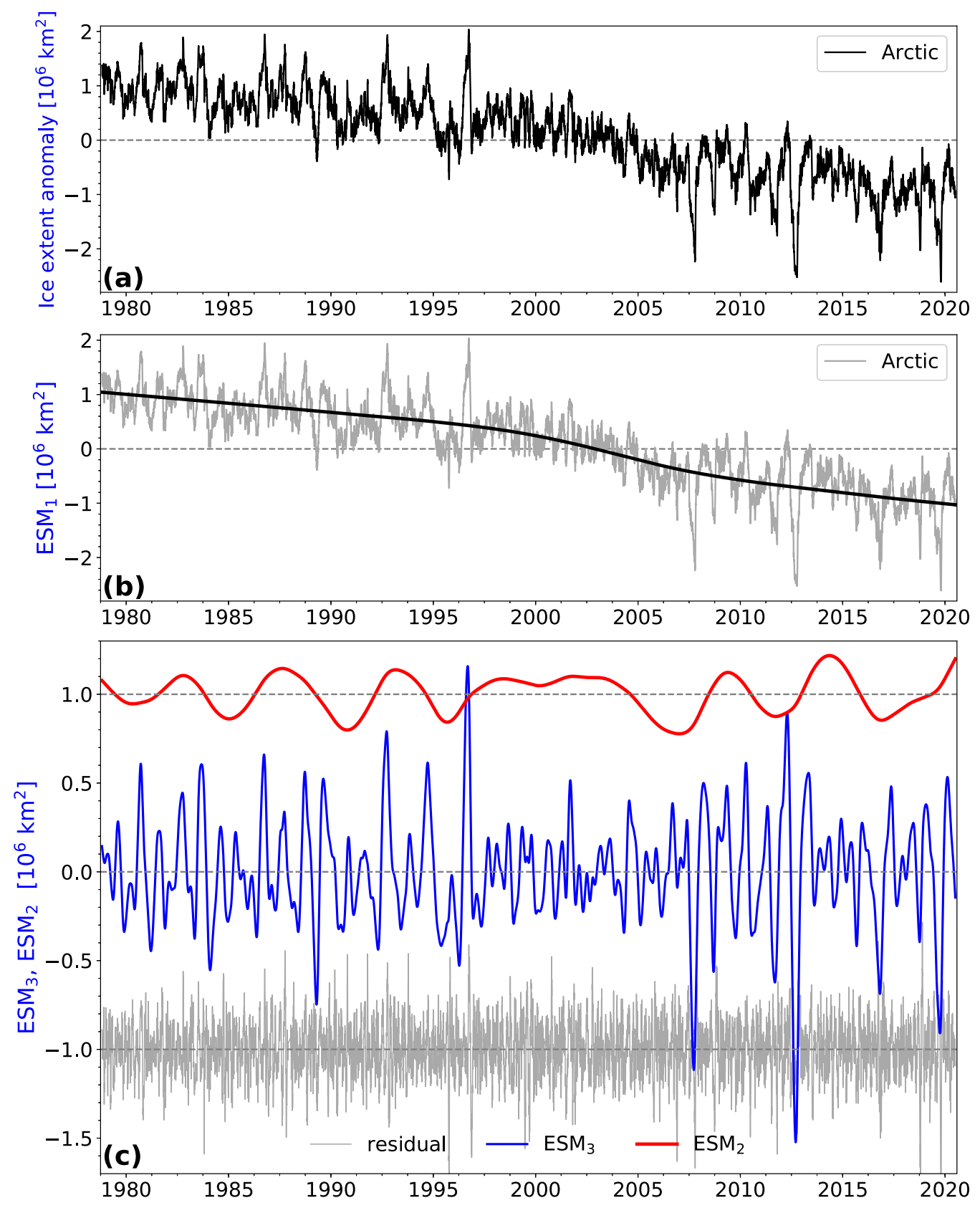

FIG. 6. Bottom-to-top decomposition of the daily ice extent anomaly time series for the Arctic. (a) Full record. (b) Background trend ESM determined by $p_{1}$ in Fig. 5(b). (c) $\mathrm{ESM}_{2}$ (red, shifted upward), $\mathrm{ESM}_{3}$ (blue), and residuals (gray, shifted downward) determined by $p_{2}$ and $p_{3}$ [see Fig. 5(b)].

melting at the northern pole. This smooth trend is the first slow mode $\mathrm{ESM}_{1}$ [Fig. 6(b)] obtained with the smoothness parameter $p_{1}$ [Fig. 5(b)]. It seems that a regime change happened between 2000 and 2005: In the last two decades of the 20th century, fluctuations exhibited large-amplitude positive (freezing) spikes, while in the first two decades of the $21 \mathrm{st}$ century the sign changed and large-amplitude negative (melting) spikes dominate.

$\mathrm{ESM}_{2}$ [red curve in Fig. 6(c)] is a slow quasiperiodic oscillatory mode with a characteristic period of around 5 years, with a visible phase shift in the early 2000s, coinciding with the suggested regime change.
$\mathrm{ESM}_{3}$ [blue curve in Fig. 6(c)] exhibits much-largeramplitude oscillations than $\mathrm{ESM}_{2}$, as expected already from the peak heights in Fig. 5(b). The dominating period is sharply 1 year and the regime change is also apparent: Large positive peaks in the 20th century are followed by dampened fluctuations in the early 2000s and large negative annual excursions are dominating in the last 15 years.

The residuals [Fig. 6(c), gray line] have a broad, verysmall-amplitude Fourier hump centered at around 80-100 days (not shown here), which can be reasonably related to short-term fluctuations of different amplitudes in the different seasons. 

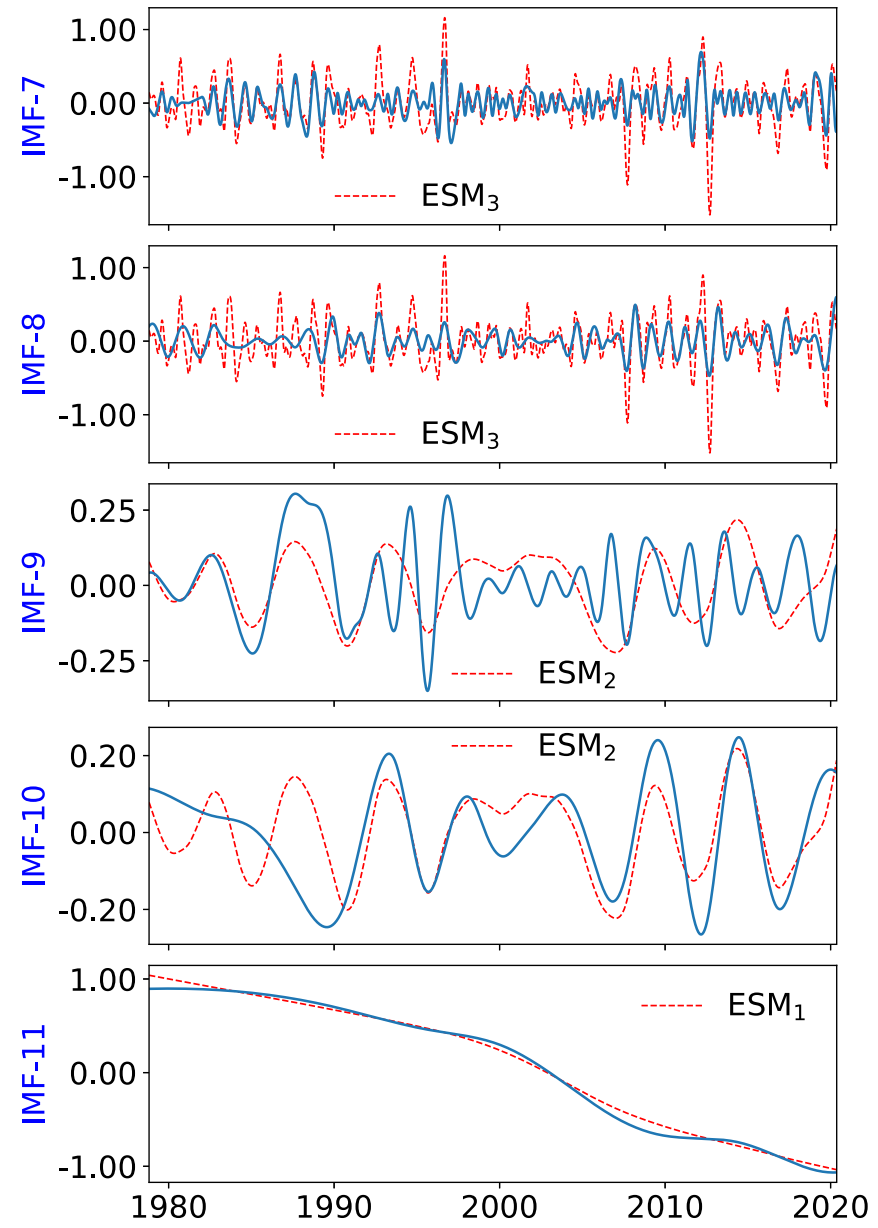

FIG. 7. Last five IMFs (blue lines) of the Arctic daily ice extent anomalies [shown in Fig. 6(a)] produced by the CEEMDAN algorithm. Red dashed lines illustrate the ESMs of approximately similar characteristic frequencies.

The comparison of our results with the top-to-bottom CEEMDAN decomposition is illustrated in Fig. 7. Similarly to the synthetic noise in Fig. 4, the algorithm produced here 11 IMFs, the first six representing high-frequency modes. A visual inspection reveals again substantial mode mixing. While IMF-7 seems quite similar to $\mathrm{ESM}_{3}$ identified in Fig. 6(c) (blue line), large discrepancies are present at the peaks of largest amplitudes. IMF-8 provides some amplitude corrections to IMF-7, although the mismatch with $\mathrm{ESM}_{3}$ is obvious. Particularly strong mode mixing is apparent when we compare IMF-9 and IMF-10 with the essential slow mode $\mathrm{ESM}_{2}$. Note that even the slow general trend of $\mathrm{ESM}_{1}$ is poorly resolved by the last intrinsic mode function IMF-11.

\section{B. Antarctic}

We repeated the same analysis for the Antarctic ice extent time series, following the steps of Sec. III A. Daily ice extent anomalies are plotted in Fig. 8(a). The slowest background mode $\mathrm{ESM}_{1}$ [Fig. 8(b)] identified by the cubic spline fit with $p_{1}$ in Fig. 5(c) is totally different from the monotonically decreasing long-term trend at the Arctic [Fig. 6(b)]. The lack of overall shrinking of the Antarctic ice sheet until around 2015
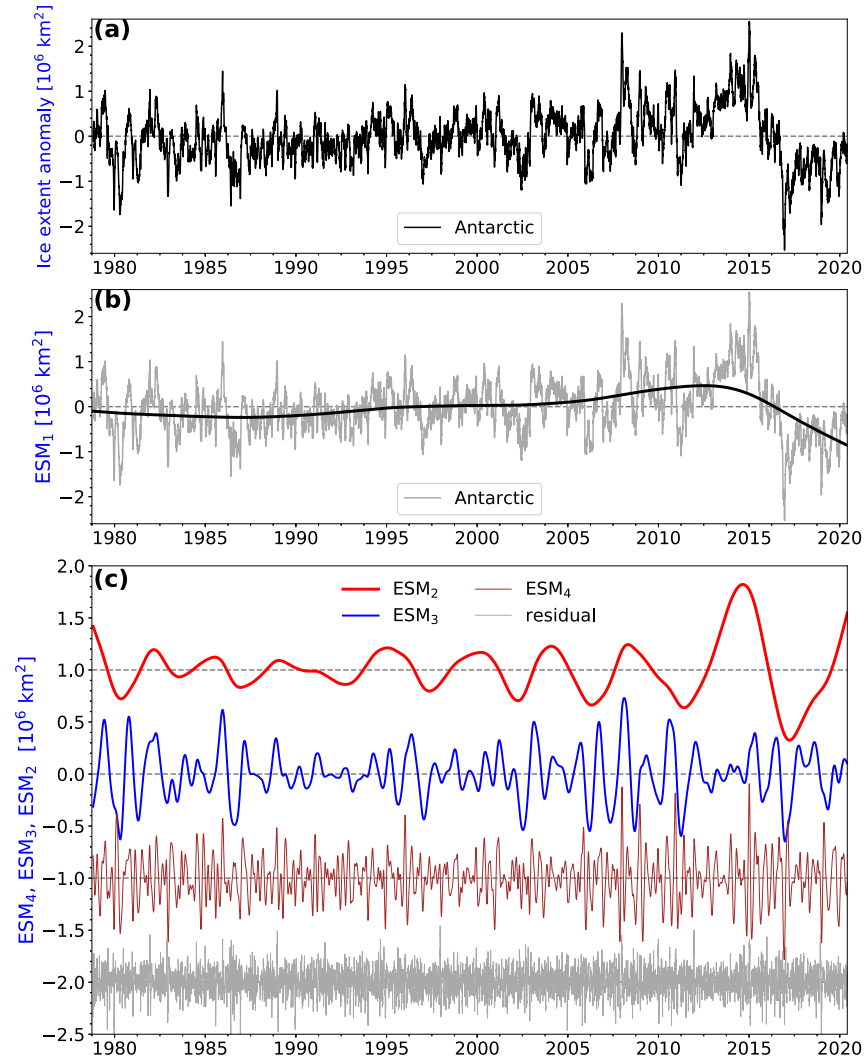

FIG. 8. Bottom-to-top decomposition of the daily ice extent anomaly time series for the Antarctic. (a) Full record. (b) Background trend $\mathrm{ESM}_{1}$ determined by $p_{1}$ in Fig. 5(d). (c) $\mathrm{ESM}_{2}$ (red, shifted upward), $\mathrm{ESM}_{3}$ (blue), $\mathrm{ESM}_{4}$ (brown, shifted downward), and residuals (gray, shifted downward) determined by $p_{2}, p_{3}$, and $p_{4}$ [see Fig. 5(d)].

is well known again, and possible explanations are widely discussed in the geophysical literature (together with the quick melting during 2015-2016).

The slowest oscillatory mode $\mathrm{ESM}_{2}$ [Fig. 8(c), red curve] seems to be similar to the Arctic 5-year mode. However, a Fourier test suggests a somewhat longer period of around 5.5 years. Note the swing of $\mathrm{ESM}_{2}$ with an increasing amplitude in the last two decades. (Since such behavior is not consistent with the stationarity criteria required by spectral analysis, we do not refer to details here.)

The next fluctuating mode $\mathrm{ESM}_{3}$ of $p_{3}$ [Fig. 8(c), blue curve] exhibits a characteristic peak at a period of 1.2-1.3 years, which is somewhat perplexing. The lack of a clear 1-year component (in strong contrast with the Arctic) might be a consequence of interferences among the annual periodicity and distinct climate modes, such as the Southern Annular Mode, quasistationary wave pattern, Pacific South American pattern, and SemiAnnual Oscillation, all known to be related to the Antarctic ice extent [44-46].

Similarly interesting is the fastest oscillatory mode $\mathrm{ESM}_{4}$ of $p_{4}$ [Fig. 8(c), brown curve]. It has a comparable amplitude to $\mathrm{ESM}_{3}$ and a broad spectral hump centered at around a period of 160-180 days, somewhat less than half a year. This is again in contrast with the Arctic signal; however, 

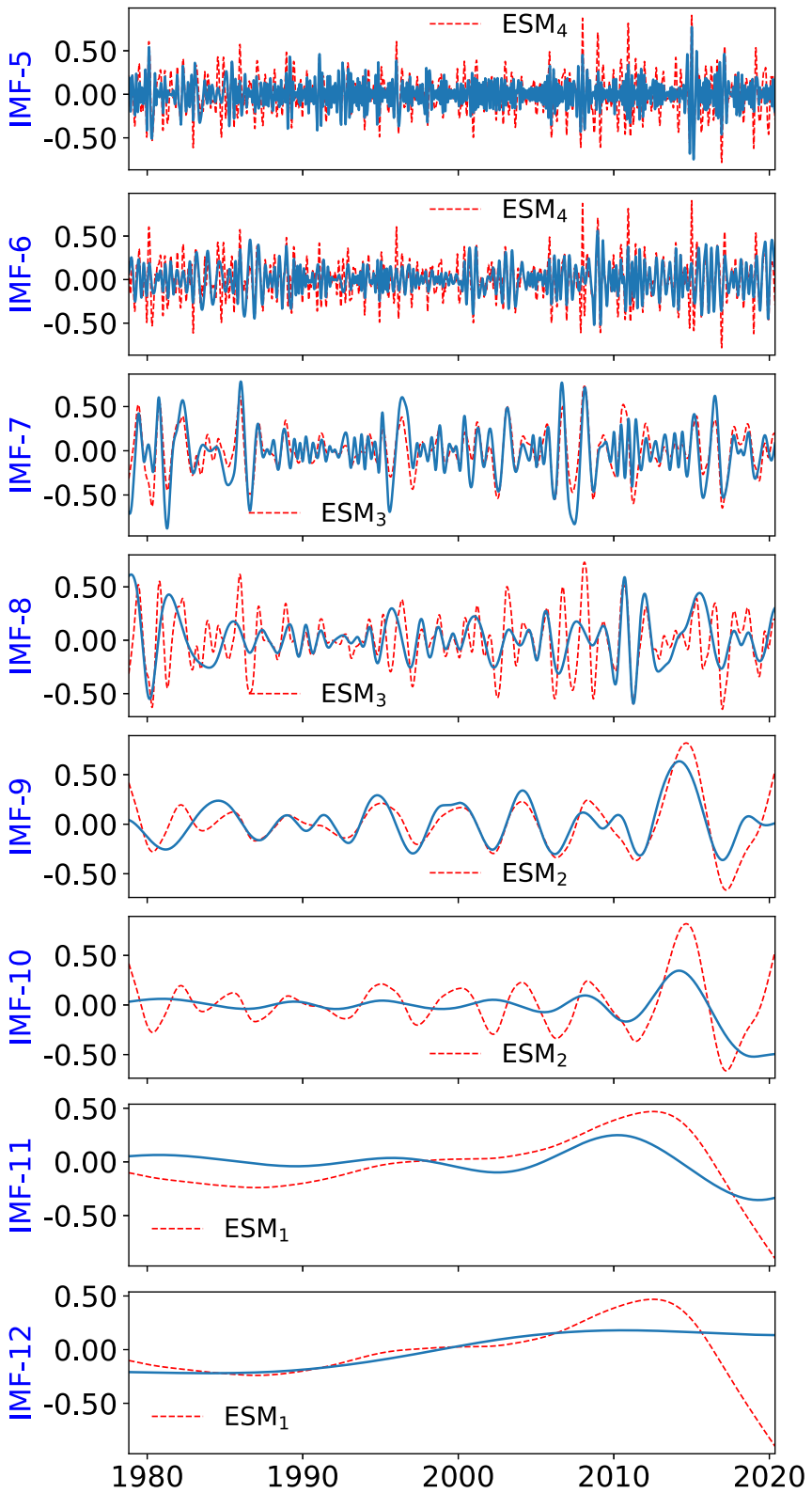

FIG. 9. Last eight IMFs (blue lines) of the Antarctic daily ice extent anomalies [shown in Fig. 6(a)] produced by the CEEMDAN algorithm. Red dashed lines illustrate the ESMs of approximately similar characteristic frequencies.

semiannual oscillations are known to determine many meteorological patterns on the southern hemisphere [44-46].

Unlike the Arctic, the Antarctic residuals [Fig. 8(c), gray line] have negligible spectral peaks at small periods between 1 and 100 days and zero for larger values. This indicates that the extra near semiannual mode $\mathrm{ESM}_{4}$ (superposed with the slower ESMs) in the case of Antarctic explains all the relevant variabilities apart from some uncorrelated random noise.

The CEEMDAN results for the Antarctic are illustrated in Fig. 9, where the first four IMFs represent noise. Again, the top-to-bottom decomposition cannot reproduce ESMs properly. While IMF-9 seems to be close to $\mathrm{ESM}_{2}$, significant discrepancies are obvious. The closest match is visible between IMF-7 and $\mathrm{ESM}_{2}$, at least for the characteristic
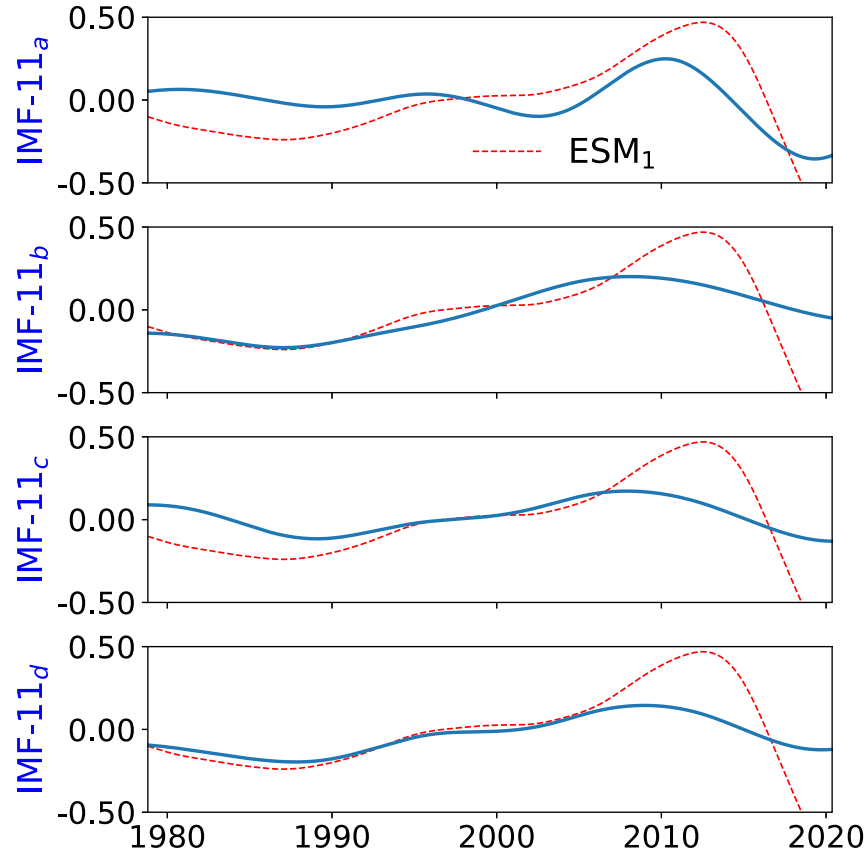

FIG. 10. The IMF-11 (blue lines) from four runs of the CEEMDAN decomposition of the Antarctic ice extent anomaly time series in Fig. 8(a). The only difference between runs is that the seed of the random number generator producing the noise is set from an internal timer. $\mathrm{ESM}_{1}$ [see Fig. 8(b)] is illustrated by red dotted lines.

frequencies, although amplitudes are overestimated by IMF-7. Notably, the long-term trend ESM 1 spectacularly suffers from mode mixing; even the sum of IMF-11 plus IMF-12 does not fit to $\mathrm{ESM}_{1}$, particularly at the ends of the signal.

\section{DISCUSSION}

While empirical methods based on mode decomposition are able to provide a kind of basis function for noisy, nonstationary, nonlinear signals, the method of top-to-bottom decomposition suffers from several shortcomings. The basic question is to interpret the IMFs: Do they represent physical modes or not? The forced dyadic filtering usually results in ten or more IMFs for empirical records; the first few are the noise parts, depending strongly on the first local maxima and minima representing the upper and lower envelopes. Noise-assisted variants like EEMD and CEEMDAN seemingly override this randomness with a decomposition of an ensemble of noise decorated records. However, Fig. 10 illustrates a peculiar feature of such procedures, namely, repeated ensemble realizations often result in strongly different IMFs.

In Fig. 10, the results for four subsequent runs of CEEMDAN decomposition are illustrated for the Antarctic ice extent anomaly time series [Fig. 8(a)]. In each run, an ensemble of 100 test sets is generated with an internal random number generator seed [38]. Particularly the low-frequency intrinsic mode functions, such as IMF-11 plotted in Fig. 10, suffer from an extreme variability, questioning any interpretation as being representative of a physical mode.

There are several proposals to identify relevant IMFs (presumably representing some physical modes) (see, 
e.g., [47-50]). The limitation of all proposals is the following. The first step always is the top-to-bottom decomposition into a limited number of IMFs and then some measures of correlation with the original signal are evaluated to discriminate relevant and irrelevant IMFs. In contrast, by evaluating hundreds of IMFs our method is able to identify ESMs which represent particularly well the overall tendencies of the signal.

In conclusion, we believe the proposed bottom-to-top cubic spline decomposition to provide a competitive tool to identify modes in strongly fluctuating data and to significantly advance the state of the art regarding decomposition of time series. The computational cost (CPU time) demanded by our algorithm with 100-200 cubic spline fits is at most one-tenth of a standard CEEMDAN procedure.

\section{ACKNOWLEDGMENTS}

This work was supported by the Max-Planck Institute for the Physics of Complex Systems in the framework of an Advanced Study Group on "Forecasting with Lyapunov vectors." I.M.J. was supported by the Hungarian National Research, Development and Innovation Office (Grants No. FK-125024 and No. K-125171). M.W.B. and J.A.C.G. were partially supported by CNPq, Brazil, Grants No. 310792/2018-5 and No. 304719/2015-3.

\section{APPENDIX: HOW DOES THE METHOD WORK?}

Since our bottom-to-top decomposition method is a fully empirical and data-driven procedure, it is impossible to provide a comprehensive exploration of the infinite variety of artificial signals. Instead, we are using simple harmonic functions and Gaussian noise as a first test:

$$
y(t)=\sin [2 \pi t /(365.25 \times T)]+A \times G(0,1) .
$$

The harmonic component has a unit amplitude, the time $t$ is measured in days, $T$ is measured in years, and $G(0,1)$ represents a Gaussian white noise of zero mean and unit standard deviation, with amplitude $A$.

In order to check the sensitivity of the procedure for the resolution of the smoothing parameter $p$, we constructed three sets covering the range $p \in\left[10^{-16}, 3 \times 10^{-3}\right]$ (with logarithmic spacing) as follows. The set $p 151$ is formed by $p 151_{n}=1.23 \times p 151_{n-1}$ for $n=2, \ldots, 151$. Similarly, p301 is constructed as $p 301_{n}=1.11 \times p 301_{n-1}$ for $n=$ $2, \ldots, 301$. Finally, $p 601$ has the rule of $p 601_{n}=1.053 \times$ $p 601_{n-1}$ for $n=2, \ldots, 601$. In all three cases the first value is $10^{-16}$.

Figure 11(a) illustrates the results for single harmonic components with a period time $T=3$ without noise, while Fig. 11(b) presents analogous data for the same sinusoidal slow mode with noise $[A=2$ in Eq. (A1)]. The shape of the $R^{2}(p)$ curves is a simple sigmoid (not shown here) starting from zero and shooting up to one with a logarithmic slope; consequently, the derivative is an almost symmetric peak on a semilogarithmic scale. (Note that on a linear scale such peaks are strongly asymmetric with typical skewness values on the order of 2.4 , almost independently of the location.) When the single peaks are normalized by their peak maxima,
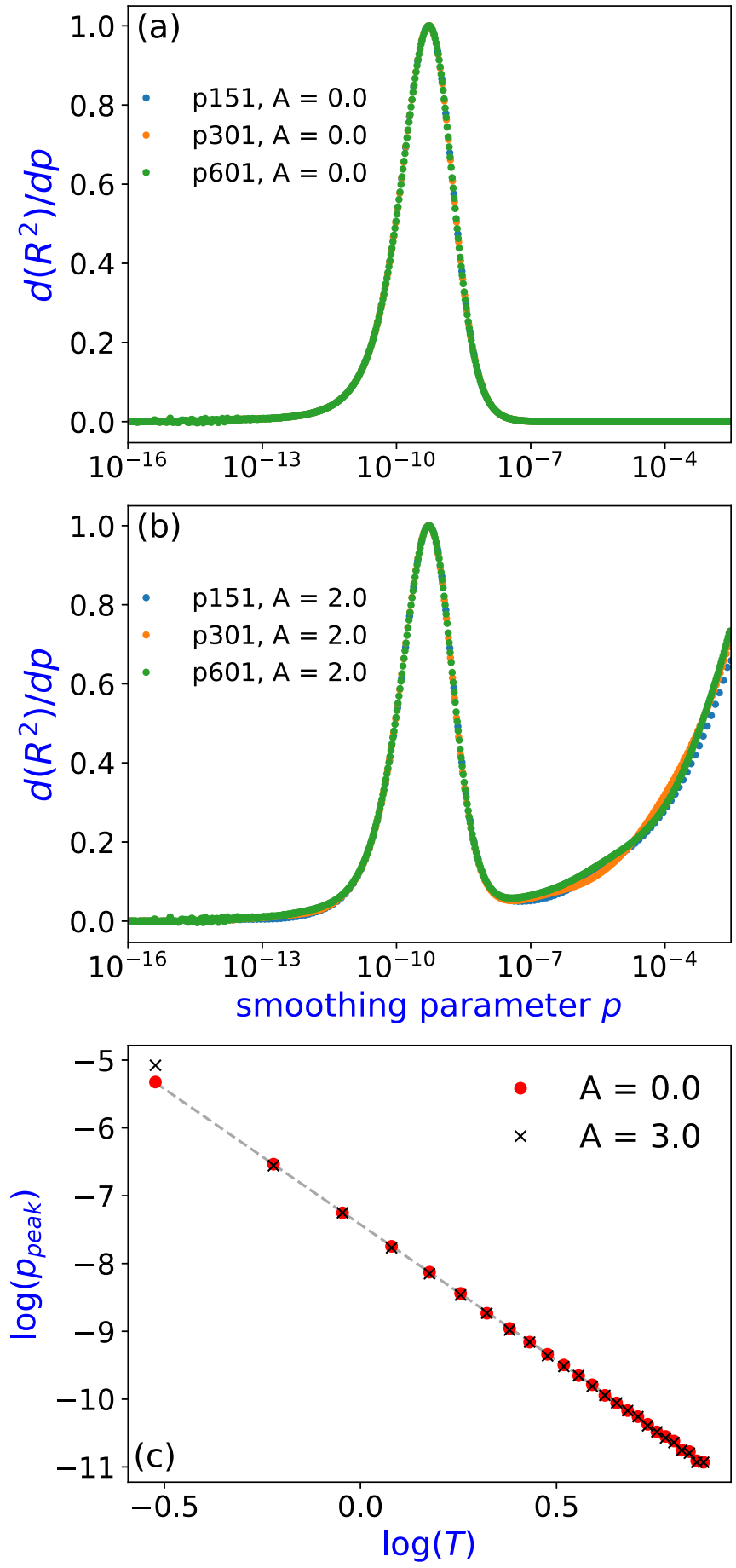

FIG. 11. (a) Gradient of the coefficient of determination $d\left(R^{2}\right) / d p$ for a pure sinusoidal signal with $T=3$ [see Eq. (A1)] with three different series of smoothing parameter $p$ defined in the text. The peaks are normalized by the maxima. (b) Same as (a) with a large noise component $[A=2$ in Eq. (A1)]. (c) Logarithm (base 10) of peak location $\log \left(p_{\text {peak }}\right)$ as a function of the logarithm of period time $\log (T)$ with zero noise (red symbols) and with a large noise ( $A=3$, black crosses). The log-log plot indicates the scaling of $p_{\text {peak }} \sim T^{-4}$ (dashed line). The smoothing parameter series $p 601$ was used in the test. 

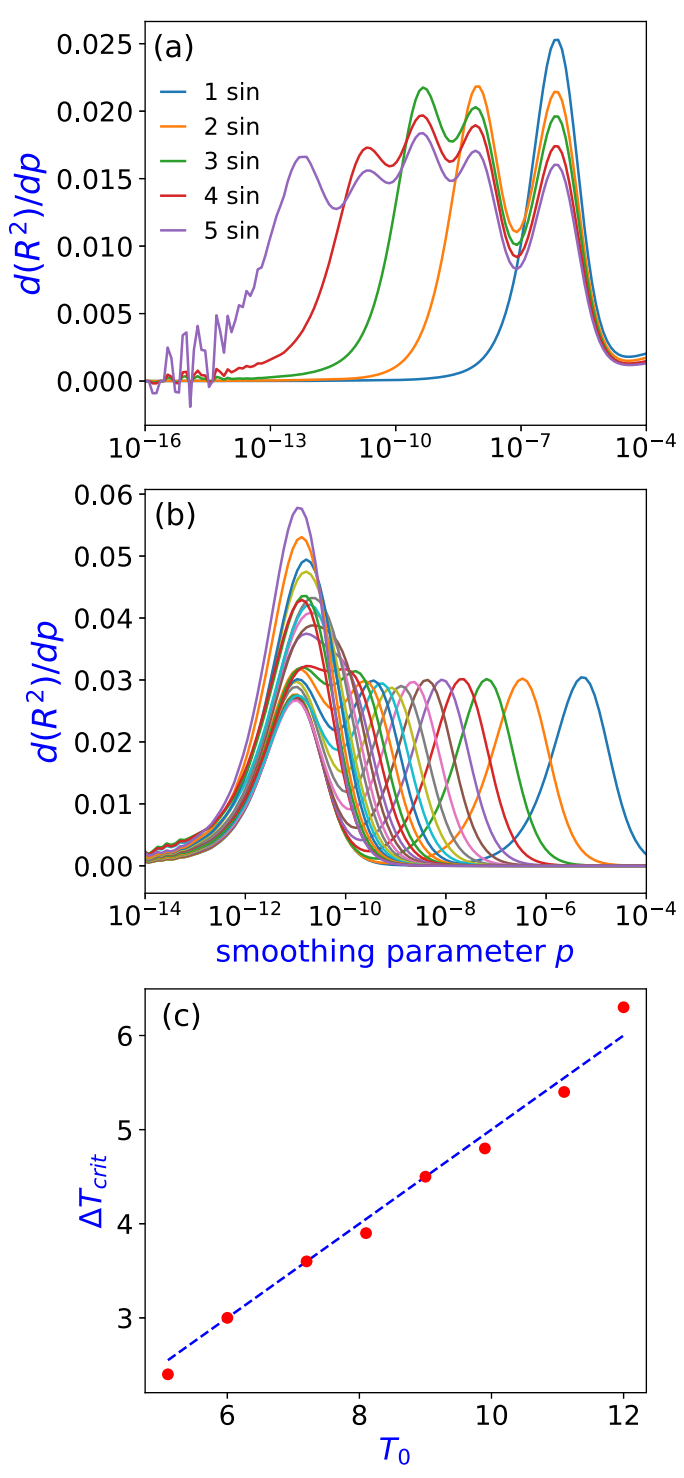

FIG. 12. (a) Gradient of the coefficient of determination $d\left(R^{2}\right) / d p$ for a superposition of up to five sinusoidal signals with $T=0.5,1.5,3.3,7.1$, and 15.3 with the $p 151$ parameter series defined in the text. Gaussian noise with $A=1$ is also added for each case. (b) Plot of $d\left(R^{2}\right) / d p$ for the superposition of two sinusoidal signal with 25 different $\Delta T$ values. There is a fixed component with $T=8.1$ (leftmost peak), and the period time of the other component increases from $T=0.3$ (rightmost peak) by steps of $T_{+}=0.3$. (c) Critical separation distance $\Delta T_{\text {crit }}$ where the higherfrequency component can be clearly identified (double peak with local minimum in between) as a function of the slow component with $T_{0}$. For comparison, the dashed line indicates the relationship of $\Delta T_{\text {crit }}=T_{0} / 2$. they fully coincide. Normalization is necessary for the comparison, because finer steps of the smoothing parameter $p$ obviously result in smaller values of the numerical gradient $d\left(R^{2}\right) / d p$. The remarkable result in Fig. 11(b) is that the procedure is weakly sensitive to the high-frequency noise and the slow sinusoidal mode is easily detectable with surprisingly large noise amplitudes without any problem (the example $A=$ 2 means twice larger noise amplitude than the slow mode). The presence of noise is visible for larger and larger smoothness parameters behind the peak and the quality of cubic spline fits improves again by progressively representing the noise better and better.

The position of the peak functionally depends on the characteristic period time, as illustrated in Fig. 11(c). The horizontal axis is $\log (T)$ (covering the interval of $[0.3,7.5]$ years by steps of 0.3 year) and the vertical axis is the position of the peak $\log \left(p_{\text {peak }}\right)$. The dashed line has a fitted slope of -4.02, indicating a power-law dependence: $p_{\text {peak }} \sim T^{-4}$.

The widths of the peaks on semilogarithmic scales [such as in Figs. 11(a) and 11(b)] are hardly dependent of the position, but the scaling behavior with $T^{-4}$ means that the separation of peaks decreases sharply at increasing $T$ values. This obviously limits the identification of slow sinusoidal components of close frequencies.

Figure 12(a) illustrates that the superposition up to five sinusoidal modes is clearly decomposable when the separation of characteristic frequencies is large enough. A feasible bottom-to-top decomposition requires clear local minima between the peaks. As expected, when the separation is too small, the peaks merge and the identification of such slow modes is not possible, as clearly visible in Fig. 12(b). The critical separation $\Delta T_{\text {crit }}$, where the double peak has a clear local minimum, depends on the period time, as noted above. We performed a series of tests similarly to the ones in Fig. 12(b) with different period time of the slower component $T_{0}$ and identified the last clear double peak as a function of separation, which defines a critical difference $\Delta T_{\text {crit }}$. Figure 12(c) illustrates the result, which suggests a surprisingly simple linear relationship $\Delta T_{\text {crit }}=T_{0} / 2$ for two sinusoidal components.

It is clear that there is a plethora of questions about the sensitivity and selectivity of the bottom-to-top decomposition, such as modes with different amplitudes, slow background trends, and signals other than sinusoidal. A detailed analysis of every such aspects is beyond the scope of this work. Nevertheless, we believe that the method works and is efficient and that it is able to identify slow nonlinear modes present in complicated signals when the condition of large enough characteristic frequency separation is fulfilled.
[1] A. Vecchio and V. Carbone, Phys. Rev. E 82, 066101 (2010).

[2] V. Capparelli, A. Vecchio, and V. Carbone, Phys. Rev. E 84, 046103 (2011).

[3] S. Dwivedi, B. N. Goswami, and F. Kucharski, Geophys. Res. Lett. 42, 8201 (2015).

[4] Y. Quilfen and B. Chapron, Geophys. Res. Lett. 46, 253 (2019).
[5] J. Huang, T. Ou, D. Chen, Y. Luo, and Z. Zhao, Geophys. Res. Lett. 46, 13338 (2019).

[6] M.-C. Wu and C.-K. Hu, Phys. Rev. E 73, 051917 (2006).

[7] M.-C. Wu, E. Watanabe, Z. R. Struzik, C.-K. $\mathrm{Hu}$, and Y. Yamamoto, Phys. Rev. E 80, 051917 (2009). 
[8] C. M. Sweeney-Reed, S. J. Nasuto, M. F. Vieira, and A. O. Andrade, Adv. Data Sci. Adapt. Anal. 10, 1840001 (2018).

[9] K. Barnova, R. Martinek, R. Jaros, and R. Kahankova, IEEE Access 8, 51200 (2020).

[10] A. Goska and A. Krawiecki, Phys. Rev. E 74, 046217 (2006).

[11] M.-T. Lo, V. Novak, C.-K. Peng, Y. Liu, and K. Hu, Phys. Rev. E 79, 061924 (2009).

[12] K. Manchanda and R. Ramaswamy, Phys. Rev. E 83, 066201 (2011).

[13] N. Nava, T. D. Matteo, and T. Aste, Physica A 502, 534 (2018).

[14] L. Yang, L. Zhao, and C. Wang, Physica A 531, 121813 (2019).

[15] B. Zhu, L. Huang, L. Yuan, S. Ye, and P. Wang, Int. Rev. Econ. Financ. 67, 163 (2020).

[16] N. E. Huang, Z. Shen, S. R. Long, M. C. Wu, H. H. Shih, Q. Zheng, N.-C. Yen, C. C. Tung, and H. H. Liu, Proc. R. Soc. London Ser. A 454, 903 (1998).

[17] N. E. Huang, in Wavelet Applications VIII, edited by H. H. Szu, D. L. Donoho, A. W. Lohmann, W. J. Campbell, and J. R. Buss (SPIE, Bellingham, 2001), Vol. 4391, pp. 71-80.

[18] N. E. Huang and Z. Wu, Rev. Geophys. 46, RG2006 (2008).

[19] Z. Wu and N. E. Huang, Adv. Adapt. Data Anal. 01, 1 (2009).

[20] M. A. Colominas, G. Schlotthauer, M. E. Torres, and P. Flandrin, Adv. Adapt. Data Anal. 04, 1250025 (2012).

[21] M. E. Torres, M. A. Colominas, G. Schlotthauer, and P. Flandrin, in Proceedings of the 2011 IEEE International Conference on Acoustics, Speech, and Signal Processing (ICASSP), Prague, 2011 (IEEE, Piscataway, 2011), pp. 4144-4147.

[22] M. A. Colominas, G. Schlotthauer, and M. E. Torres, Biomed. Signal Proces. Control 14, 19 (2014).

[23] Y. Gao, G. Ge, Z. Sheng, and E. Sang, Proceedings of the 2008 Congress on Image and Signal Processing, Sanya, 2008 (IEEE, Piscataway, 2008), Vol. 5, pp. 223-227.

[24] X. Hu, S. Peng, and W. Hwang, IEEE Trans. Signal Process. 60, 1075 (2012).

[25] J. Zheng, J. Cheng, and Y. Yang, Signal Process. 96, 362 (2014).

[26] I. M. Jánosi and R. Müller, Phys. Rev. E 71, 056126 (2005).

[27] P. Flandrin, G. Rilling, and P. Gonçalves, IEEE Signal Process. Lett. 11, 112 (2004).

[28] Z. Wu and N. E. Huang, Proc. R. Soc. A 460, 1597 (2004).

[29] N. E. Huang and S. S. P. Shen, Hilbert-Huang Transform and its Applications, 2nd ed. (World Scientific, Singapore, 2014).
[30] F. Fetterer, K. Knowles, W. N. Meier, M. Savoie, and A. K Windnagel, Sea Ice Index, version 3, https://doi.org/10.7265/ N5K072F8 (National Snow and Ice Data Center, Boulder, 2017), updated daily; accessed 22 May 2020.

[31] WMO Guidelines on the Calculation of Climate Normals, https: //library.wmo.int/index.php (WMO, Geneva, 2017), No. 1203.

[32] E. Prilepin, CSAPS: Cubic Spline Approximation, https://pypi. org/project/csaps/ (Python Software Foundation, Fredericksburg, 2019).

[33] G. Wahba and S. Wold, Commun. Statist. 4, 1 (1975).

[34] T. Robinson and R. Moyeed, Commun. Stat. 18, 523 (1989).

[35] P. H. C. Eilers and B. D. Marx, Stat. Sci. 11, 89 (1996).

[36] S. Arlot and A. Celisse, Stat. Surv. 4, 40 (2010).

[37] D. R. Legates and G. J. McCabe, Jr., Water Resour. Res. 35, 233 (1999).

[38] D. Laszuk, Python implementation of empirical mode decomposition algorithm, http://www.laszukdawid.com/codes (2017).

[39] R. Sharma, L. Vignolo, G. Schlotthauer, M. Colominas, H. L. Rufiner, and S. Prasanna, Speech Commun. 88, 39 (2017).

[40] Z. Qian, Y. Pei, H. Zareipour, and N. Chen, Appl. Energ. 235, 939 (2019).

[41] S. Chen, X. Dong, Z. Peng, W. Zhang, and G. Meng, IEEE Trans. Signal Process. 65, 6024 (2017).

[42] M. Nazari and S. M. Sakhaei, Signal Process. 174, 107610 (2020).

[43] G. Tang and H. Yang, Multiscale Model. Simul. 18, 707 (2020).

[44] X. Yuan and C. Li, J. Geophys. Res.: Oceans 113, C06S91 (2008).

[45] M. Van Den Broeke, Int. J. Climatol. 20, 455 (2000).

[46] C. Eayrs, D. Holland, D. Francis, T. Wagner, R. Kumar, and X. Li, Rev. Geophys. 57, 1037 (2019).

[47] Z. Peng, P. W. Tse, and F. Chu, Mech. Syst. Signal Process. 19, 974 (2005).

[48] A. Ayenu-Prah and N. Attoh-Okine, Adv. Adapt. Data Anal. 02, 1 (2010).

[49] D. B. de Souza, J. Chanussot, and A. Favre, in Proceedings of the IEEE International Conference on Acoustics, Speech, and Signal Processing (ICASSP), Florence, 2014 (IEEE, Piscataway, 2014), pp. 325-329.

[50] S. Kotan, J. Van Schependom, G. Nagels, and A. Akan, in Proceedings of the 2019 Medical Technologies Congress (TIPTEKNO), Izmir, Turkey, 2019 (IEEE, Piscataway, 2019), pp. 1-4. 